\title{
Quality of Life among Persons Aged 60-84 Years in Europe: The Role of Psychological Abuse and Socio-Demographic, Social and Health Factors
}

Joaquim JF Soares $^{1 *}$, Örjan Sundin ${ }^{2}$, Eija Viitasara', Maria Gabriella Melchiorre ${ }^{3}$, Mindaugas Stankunas ${ }^{4,5}$, Jutta Lindert ${ }^{6}$, Francisco TorresGonzales $^{7}$, Henrique Barros ${ }^{8}$ and Elisabeth loannidi-Kapolou ${ }^{9}$

${ }^{1}$ Department of Public Health Sciences, Mid Sweden University, Sundsvall, Sweden

${ }^{2}$ Department of Psychology, Mid Sweden University, Östersund, Sweden

3/talian National Institute of Health and Science on Aging, INRCA, Ancona, Italy

${ }^{4}$ Department of Health Management, Lithuanian University of Health Sciences, Kaunas, Lithuania

${ }^{5}$ School of Public Health, Griffith University, Gold Coast Campus, Queensland, Australia

${ }^{6}$ Department of Public Health Science, Protestant University of Applied Sciences, Ludwigsburg, Germany

${ }^{7}$ Network of Biomedical Research on Mental Health Centers, University of Granada, Spain

${ }^{8}$ Department of Hygiene and Epidemiology, University of Porto, Medical School, Porto, Portugal

${ }^{9}$ Department of Sociology, National School of Public Health, Athens, Greece

\begin{abstract}
Background: Elder abuse and its effects are a serious public health issue. However, little is known about the relation between psychological abuse, other factors (e.g. social support) and quality of life (QoL) by domain. This study addressed differences in QoL by domain between psychologically abused and non-abused. While considering other factors such as social support.

Methods: The respondents were 4,467 (2,559 women) randomly selected persons aged 60-84 years living in 7 European cities. The mean response across countries was $45.2 \%$. The cross-sectional data were analyzed with bivariate/multivariate methods.

Results: Abused respondents contrasted to non-abused scored lower in QoL (autonomy, $67.42 \pm 21.26$ vs. 72.39 \pm 19.58; intimacy, $55.31 \pm 31.15$ vs. $67.21 \pm 28.55$; past/present/future activities, $62.79 \pm 19.62$ vs. $68.05 \pm 18.09$; social participation, $65.03 \pm 19.84$ vs. $68.21 \pm 19.77$ ). Regressions showed that abuse was negatively associated with autonomy, intimacy and past/present/future activities, and positively with the social participation. All QoL dimensions were negatively associated with country and depressive/anxiety symptoms, and positively with social support. Further, variables such as age, sex and somatic symptoms were negatively associated with some of the QoL dimensions and others such as family structure, education, health care use and drinking positively. The regression model "explained" $32.8 \%$ of the variation in autonomy, $45.6 \%$ in intimacy, $44.8 \%$ in past/present/future activities and $41.5 \%$ in social participation.

Conclusions: Abuse was linked to lower QoL in most domains, but other factors such as depressive symptoms also carried a negative impact. Social support and to some extent family structure had a "protective" effect on QoL. Abuse, health indicators (e.g. depressive symptoms) and social support should be considered in addressing the QoL of older persons. However, QoL was influenced by many factors, which could not be firmly disentangled due to the crosssectional approach, calling for longitudinal research to address causality.
\end{abstract}

\section{Keywords:}

\section{Background}

Elder abuse is acknowledged as a public health problem and generates increasing concern specially as current projections indicate that persons aged 60-plus will increase from 760 million 2011 to 2 billion by 2050 [1-3]. Prevalence rates of elder abuse vary contingent on various factors such as operational definition of abuse and type of sample. For instance, a review of 49 studies reported a mean elder abuse rate of $13 \%$, and rates in the general population varied between $3.2-27.5 \%$ and over $6 \%$ had been abused during the last month [4]. Recent studies in the USA, Israel and Europe with general population/community samples observed abuse rates ranging between $0.6-27.1 \%$ depending on the type [5-7]. Among selected samples such as older persons living in a residence home, abuse rates up to $55 \%$ depending on the type have been reported [4]. Elder abuse, not least physical, co-exists with detrimental effects such as depression, premature death and reduced social support [5,8-16]. Psychological abuse, e.g. being excluded and repeatedly ignored, seems to be the most commonly reported by elders, with prevalence rates up to $52 \%[4,5,7]$. In a recent WHO report [3], it was estimated that 29 million of persons aged 60 years and over are psychologically abused each year in Europe. Psychological abuse often co-occurs with other abuse categories, e.g. financial $[5,17,18]$ and has been associated with poor health, e.g. trauma [5,19-22]. Further, psychological abuse may be more damaging for older persons than other abuse types $[5,23]$.

Various factors such as poor mental health and lack of social support have been connected with decreased quality of life (QoL) among older

${ }^{*}$ Corresponding author: Joaquim JF Soares, Institution for Health Sciences, Department of Public Health Sciences, Mid Sweden University, Holmgatan 10, Humlegården, Hus M, 85170 Sundsvall, Sweden, Tel: +46 (0) 601485 03; E-mail: joaquim.soares@miun.se

Received January 18, 2013; Accepted March 20, 2013; Published March 23, 2013

Citation: Soares JJF, Sundin Ö, Viitasara E, Melchiorre MG, Stankunas M, et al. (2013) Quality of Life among Persons Aged 60-84 Years in Europe: The Role of Psychological Abuse and Socio-Demographic, Social and Health Factors. J Biosafety Health Educ 1: 101. doi:10.4172/2332-0893.1000101

Copyright: (c) 2013 Soares JJF, et al. This is an open-access article distributed under the terms of the Creative Commons Attribution License, which permits unrestricted use, distribution, and reproduction in any medium, provided the original author and source are credited. 
persons [e.g. 24-34] and abuse may also be an important contributor to it. Yet, the linkage between abuse, perceived QoL and other factors such as depressive symptoms among older persons has not attracted great attention. To our knowledge, only two studies have investigated this issue [35,36]. Although both reported a relation between abuse/ neglect and decreased QoL, they differed substantially. For instance, the operational definition of abuse/neglect and what is meant with QoL is unclear in one of the studies [35]. In the other study [36], there is an unusual operational definition of for instance psychological abuse, e.g. an act of psychological abuse was considered only if it occurred 10 or more times, suggesting under estimation of abuse.

A further examination of the influence of abuse (particularly psychological), while considering other factors such as depressive symptoms upon perceived QoL by domain among older adults from the general population may be useful in several ways. First, by addressing some of the limitations of available studies as described above, we may provide a more accurate description of the relationship between abuse, other factors such as depressive symptoms and QoL among older persons. Second, as far as we know, there are no studies about the relationship between psychological abuse, other factors such as depressive symptoms and QoL with general population samples of older adults. Third, abused older persons in many countries such as Spain, Portugal and Lithuania are not being systematically and consistently assisted, although the importance of prevention has been stressed $[1,2]$. Therefore, by exploring the relationship between psychological abuse, other factors such as depressive symptoms and QoL among older persons from different countries (e.g. Spain, Portugal, Lithuania), we may provide reliable data on their experiences that could support policy makers and health planers/providers in the development of effective interventions for targeting abuse and improving QoL. Finally, limited attention has been paid to the identification of correlates of QoL (subjective) among older adults, particularly regarding the role of psychological abuse. Considering factors such as depressive symptoms may help to better understand the actual contribution of psychological abuse towards "explaining" the variation in QoL, not least as psychological abuse may be the most commonly reported form of abuse by older persons and more damaging that other abuse forms. Thus, our main aims were to: (i) Compare the subjective experience of QoL by domain (autonomy, fear of death/dying, intimacy, past/present/ future activities, sensory-abilities, social participation) between psychologically abused and non-abused persons aged 60-84 years; and (ii) Scrutinize, among all respondents, the association between psychological abuse, other factors such as depressive symptoms and QoL by domain.

\section{Methods}

\section{Respondents}

The respondents were randomly selected women/men from the general population living in 7 European cities (Stuttgart; Athens; Ancona; Kaunas; Porto; Granada; Stockholm) that took part in the survey "Elder abuse: A multinational prevalence survey, ABUEL" during January-July 2009. Inclusion criteria were age 60-84 years, no cognitive problems (e.g. dementia) [1] or sensory impairments (e.g. blindness), national citizens or documented migrants, living within the community (own/rented houses) or homes for elderly and proficiency in the native languages.

The sample size was estimated on based on municipal census (number of women/men aged 60-84 years) in each city and expected abuse prevalence ranges. Departing from an abuse prevalence of
$13 \%$, with a precision of 2.6 percent derived from a recent review [4], a sample size of 633 persons in each city was necessary. Overall, the sample consisted of 4,467 respondents (2,559 women, $57.3 \%$ ) and mean response across countries was $45.2 \%$. Details regarding for instance the target population, sampling procedures, completion rates, refusal rates and differences between countries are reported in a separate ABUEL method paper by Lindert et al. [37]. Data on demographics/socioeconomics are shown in Lindert et al. [37] and Macassa et al. [7].

\section{Definition of quality of life}

The definition of QoL proposed by the WHOQOL group was used in this study: "An individuals' perceptions of their position in life in the context of the culture and value systems in which they live, and in relation to their goals, expectations, standards and concerns. It is a broad ranging concept affected in a complex way by the persons' physical health, psychological state, level of independence, social relationships and their relationship to salient features of their environment" [38-40].

\section{Measures}

Quality of Life (QoL) was assessed with the WHOQOL-OLD [38]. It contains 24 items graded 1-5 (e.g. not at all-extremely), but after transformation scores range from 0-100 [41]. The items can be summed into a total QoL and divided into 6 domains with four items in each, i.e. autonomy (e.g. freedom to make own decisions); fear of death/dying (e.g. scared of dying); intimacy (e.g. feel a sense of companionship in life); past/present/future activities (e.g. satisfied with achievements); sensory-abilities (e.g. loss of sensory abilities affecting participation in activities); and social participation (e.g. have enough to do each day). High scores correspond to high QoL. Cronbach $\alpha$ (standardized items) for QoL across the included countries was 0.92 .

Abuse was assessed with 52 items based on the Conflict Tactic Scales 2 [42] and the UK survey of abuse/neglect of older people [36]. The items were arranged in 5 abuse sub-scales, i.e. psychological, physical, sexual, injury and financial. Additionally, neglect was assessed with 13 items (e.g. routine housework) and data were collected on other factors such as the perpetrators age and sex. The abuse acts may have occurred (how often) once, twice, 3-5, 6-10, 11-20 or $>20$ times during the past year (chronicity), did not occurred the past year, but before or never occurred. In this study, only the responses regarding psychological abuse (11 items, e.g. threatened to hit or throw something at you) during the past year were analysed. If respondents answered that abuse had not occurred during the past year, they were considered as no abuse cases (no). If respondents answered that they had been abused during the past year independently of chronicity, they were considered as abuse cases (yes). Cronbach $a$ (standardized items) for psychological abuse across the includedcountries was 0.85 .

Somatic symptoms were assessed with the short version of the Giessen Complaint List [43]. It contains 24 items graded 0-4 (not affected-very affected). The items can be summed into a total somatic symptoms and arranged in 4 domains with 6 items in each: exhaustion (e.g. tiredness); gastrointestinal (e.g. nausea); musculoskeletal (e.g. pains in joints or limbs); and heart distress (e.g. heavy, rapid or irregular heart-throbbing). The total score for all items is 96 and 24 for each symptom domain. The higher the scores, the more one is affected. Cronbach $\alpha$ (standardized items) for somatic symptoms across the included countries was 0.92 .

Depressive and anxiety symptoms were assessed with Hospital Anxiety and Depression Scale [44]. It contains 14 items graded 0-3 (e.g. not at all-most of the time), of which 7 pertain to depression (e.g. I 
feel as if I am slowed down) and 7 to anxiety (e.g. I get sudden feelings of panic). The total score for depression and anxiety is 21 each. No cases correspond to a score of 0-7, possibly cases to 8-10 and probable cases to 11-21. High scores correspond to high depression and anxiety levels. Cronbach a (standardized items) for anxiety across the included countries was 0.81 and for depression 0.80 .

Health care use was assessed as the number of contacts with health care staff (e.g. physician) and health care services (e.g. primary care). We also assessed the number of diseases (e.g. cardiovascular), which the respondents suffered from presently. The items were derived from the Stockholm County Council health survey [45].

Social support was assessed with the Multidimensional Scale of Perceived Social Support [46]. It contains 12 items graded 1-7 (very strongly disagree-very strongly agree). The items can be summed into a total social support and divided into 3 domains with 4 items in each: support from family (e.g. my family really tries to help me); significant others (e.g. there is a special person who is around when I am in need); and friends (e.g. I can talk about my problems with my friends). The total score ranges from 12-84, 4-28 for each domain. High scores correspond to high social support. Cronbach a (standardized items) for social support across the included countries was 0.92 .

Life-style variables were assessed as alcohol and cigarette use, and body mass index (BMI). Alcohol was assessed with items derived from The Alcohol Use Disorders Identification Test [47]. First, the respondents were asked if they presently used alcohol (do you drink alcohol? yes/no). If they answered yes, 3 items derived from Audit were applied: (1) how often do you have a drink containing alcohol? (once a month or less, 2-4 times a month, 2-3 times a week, 4 or more times a week); (2) how many drinks containing alcohol do you have on a typical day when you are drinking? ( 1 or 2,3 or 4,5 or $6,7,8$, or 9,10 or more); (3) how often do you have six or more drinks on one occasion? (Never, less than monthly, monthly, weekly, daily or almost daily). Finally, we asked respondents about their past use of alcohol (if you do not drink alcohol now, have you ever been drinking alcohol? yes/no). Smoking was assessed in a similar way. This study focused on use of alcohol and cigarettes in a yes/no format. A BMI, based on self-reported height and weight, was calculated for each respondent with the formula $\mathrm{kg} / \mathrm{m}^{2}$ (Mean/SD, 26.68/4.19; CI95\% 26.55-26.80).

Demographic/socio-economic and household variables were assessed, i.e. country, age, sex, marital status, ethnic background, education, profession, financial support, financial strain, housing, living situation, household size and if still on work. Financial strain (worries with how to make ends meet) was measured with one item (no/sometimes/often/always format). A participant was defined as having "financial strain" if she/he chose any response other than no. Four items (e.g. place of birth) assessed whether the respondents were migrants or native inhabitants. These factors were tailored for each country, but similar in content.

\section{Design/Procedure}

The design was cross-sectional. The recruitment of respondents and data gathering were conducted during January-July 2009. The data were collected via face-to-face interviews or a combination of interviews and self-response ${ }^{1}$. The scales (if not available) were translated into each country's language, back-translated and culturally adapted. Only GBB, the Multidimensional Scale of Perceived Social Support and health questions were applied the above mentioned procedure based on previously defined protocol for some of the countries. A similar strategy was applied for other materials such as information letters. Interviewers in each city $(\mathrm{n}=5-20)$ received training in various issues (e.g. ethical behavior). The respondents were carefully informed about the research in writing/verbally, and signed a consent form. Great emphasis was put on confidentiality, anonymity and the respondent's rights ${ }^{1}$. Ethical permission was received in each participating country. For further details on design/procedure see Lindert et al. [37].

\section{Statistical analyses}

Differences in QoL by domain between psychologically abused and non-abused respondents were analyzed with ANOVAs. A significant level of $\mathrm{P}<0.05$ was accepted for bivariate and multivariate analyses. Differences between psychologically abused and non-abused respondents in various areas such as demographics/socio-economics are shown elsewhere [7,37]. Additionally, we conducted collinearity statistics on the regressions, with VIF's for autonomy ranging from 1.085 to 8.552 ; for past/present/future activities from 1.067 to 7.079 ; for social participation from 1.066 to 9.596 ; and for intimacy from 1.066 to 8.943. The VIF's were below accepted levels, up to 10 (detailed data not shown here). Furthermore, Pearson correlation analyses showed positive correlation coefficients ranging from 0.000 to 0.501 and negative from -0.001 to -0.584 . The overwhelming majority of negative/ positive correlations were below 0.20 .

Further, 4 multiple linear regressions were computed to scrutinize the associations between the dependent variables (autonomy, intimacy, past/present/future activities, and social participation) and other covariates (independent variables) among all respondents ${ }^{2}$. The independent variables were selected based on statistical inference, i.e. factors such as socio-economics that differentiated abused/nonabused respondents in previous analyses $[7,37]$ and the literature on QoL. The independent variables were psychological abuse, country, age, sex, marital status, migrant background, living situation, housing, education, profession, financial support, still work, financial strain, alcohol and cigarette use, household size, BMI, health care use, diseases number, anxiety, depression, somatic symptoms and social support. Associations between the variables were expressed as unstandardized Betas/Std.Error, standardized Betas, CI95\% and R².

\section{Results}

\section{QoL and psychological abuse}

As shown in table 1, psychologically abused respondents contrasted to non-abused scored lower in autonomy, intimacy, past/present/future activities and social participation, indicating that they experienced

${ }^{1}$ Respondents who did not want to be interviewed could self-respond and a questionnaire was sent to their homes together with other materials such as information/ instruction sheets, consent letter and the Mini-Cog (Borson S, Scanlan JM, Brush M, Vitaliano PP, Dokmak A (2000) The Mini-Cog: a cognitive 'vital signs' measure for dementia screening in multi-lingual elderly. Int J Geriatr Psychiatry 15: 1021-1027). It should also be stressed, as requested by all ethical committees, that respondents were contacted by letter and telephone prior to the investigation and independently of how the data was collected form, could stop participation at any moment and call the interviewers for additionally information if necessary. Further information was provided before and under the interviews. The self-response percentages were $38 \%$ for Germany, $0.5 \%$ for Greece, $0 \%$ for Italy and Spain, $24.8 \%$ for Lithuania, $2.3 \%$ for Portugal and $63.9 \%$ for Sweden.

${ }^{2}$ Regressions of the sensory abilities and fear of death/dying categories were not performed as there were no significant differences between abused and non-abused respondents. 
Citation: Soares JJF, Sundin Ö, Viitasara E, Melchiorre MG, Stankunas M, et al. (2013) Quality of Life among Persons Aged 60-84 Years in Europe: The Role of Psychological Abuse and Socio-Demographic, Social and Health Factors. J Biosafety Health Educ 1: 101. doi:10.4172/23320893.1000101

Page 4 of 12

their QoL in these areas as lower. There were no differences regarding fear of death/dying and sensory abilities (Table 1).

\section{Correlates of QoL by domain}

As shown in table 2, autonomy was positively associated with all education levels (low, secondary, university), alcohol use and social support. Being from any other country than Germany, aged 80-84 years, financially strained and financially supported by social/sickness benefits/other pension benefits (e.g. sick pension), and experiencing anxiety, depressive andsomatic symptoms and abuse was negatively associated with autonomy. The model "explained" $32.8 \%$ of the variation in autonomy.

Past/present/future activities were positively associated with social support. Being from any other country than Germany, financially strained and financially supported by social/sickness benefits/ other pension benefits (e.g. sick pension), and experiencing anxiety, depressive and somatic symptoms and abuse was negatively associated with past/present/future activities. The model "explained" $44.8 \%$ of the variation in activities (Table 2 ).

As shown in table 3, social participation was positively associated with still on work, alcohol use, social support and abuse. Being from any other country than Germany, aged $80-84$ years, man and financially supported by social/sickness benefits/other pension benefits (e.g. sick pension), smoking, and experiencing anxiety, depressive and somatic symptoms were negatively associated with social participation. The model "explained" $41.5 \%$ of the variation in social participation.

Intimacy was positively associated with being married/cohabitant, financially strained and financially supported by spouse/partner income, living with spouse/partner, spouse/partner/other ${ }^{3}$, other persons ${ }^{4}$ and in large households, using health care frequently and social support. Being from any other country than Germany, and experiencing anxiety and depressive symptoms and abuse were negatively associated with intimacy. The model "explained" $45.6 \%$ of the variation in intimacy (Table 3).

\begin{tabular}{|c|c|c|c|}
\hline Variables & $\begin{array}{c}\text { Abused } \\
n\end{array}$ & $\begin{array}{c}\text { Not -abused } \\
n\end{array}$ & Anova \\
\hline Autonomy ${ }^{b}$ & 873 & 3505 & $(F(1,4376)=43.66, p<0.0001)$ \\
\hline Mean \pm SD & $67.42 \pm 21.26$ & $72.39 \pm 19.58$ & \\
\hline $\begin{array}{l}\text { Fear of death/ } \\
\text { dying }^{\text {b }}\end{array}$ & 871 & 3475 & $(F(1,4344)=1.38, p=0.2408)$ \\
\hline Mean \pm SD & $60.92 \pm 26.75$ & $62.17 \pm 28.47$ & \\
\hline Intimacy ${ }^{b}$ & 870 & 3521 & $(F(1,4389)=116.84, \mathrm{p}<0.0001)$ \\
\hline Mean \pm SD & $55.31 \pm 31.15$ & $67.21 \pm 28.55$ & \\
\hline $\begin{array}{l}\text { Past/present/ } \\
\text { future activities }\end{array}$ & 876 & 3534 & $(F(1,4408)=57.24, p<0.0001)$ \\
\hline Mean \pm SD & $62.79 \pm 19.62$ & $68.05 \pm 18.09$ & \\
\hline $\begin{array}{l}\text { Sensory- } \\
\text { abilities }^{b}\end{array}$ & 880 & 3547 & $(F(1,4425)=0.12, p=0.9121)$ \\
\hline Mean \pm SD & $73.42 \pm 24.54$ & $73.31 \pm 26.66$ & \\
\hline $\begin{array}{l}\text { Social } \\
\text { participation }\end{array}$ & 874 & 3537 & $(F(1,4409)=8.54, p=0.0035)$ \\
\hline Mean \pm SD & $65.03 \pm 19.84$ & $68.21 \pm 19.77$ & \\
\hline
\end{tabular}

$\mathrm{a}=$ WHOQOL-OLD=World Health Organization Quality of Life-Old; ${ }^{b}=$ sub-scales, 0-100 each one.

Table 1: Means/SD of quality of life ${ }^{a}$ by psychological abuse.

\section{Discussion}

\section{QoL and psychological abuse}

Psychologically abuse was related to lower scores in autonomy, intimacy, past/present/future activities and social participation. There were no differences between abused and non-abused respondents in fear of death/dying and sensory abilities, which may have been more affected by the ageing process than abuse. Subsequent regressions confirmed that abuse was negatively associated with autonomy, intimacy and past/present/future activities, and positively with social participation.

Psychological abuse consists of acts such as being undermined and belittled over one's activities, excluded and repeatedly ignored and prevented from seeing others. This seems contrary to QoL goals and expectations such as freedom to make own decisions (autonomy), being able to have personal and intimate relationships (intimacy), satisfaction with achievements in life (past/present/future activities) and opportunity to participate in community activities (social participation).

An explanation could be that the abuse led to feelings of worthlessness, powerlessness, hopelessness, unhappiness and insecurity. Over time these feelings may have resulted, for instance, in experiences of not being able to make own decisions, a sense that companionship with a partner or other close person was not shared to the extent desired and doubts over achievements. Elder abuse has been previously associated with poor self-esteem and unhappiness [48-50], and psychological abuse may be more damaging than other forms of abuse [5,23]. The negative effects of abuse could have been strengthened as the main perpetrators were spouses/partners (37.1\%) and significant other, e.g. offspring (34.1\%). Older persons often rely on spouses/ partners and/or significant other for assistance with daily activities, provide affection, care for their health, and may be the main source of personal care and well-being [51,52]. Being abused by near one's may have highly stressful for the older persons, for instance, in terms of effects on intimacy. It is also possible, in view of the respondents situation in other areas such as mental health problems and financial strain that for example the spouses/partners and/or significant other felt highly exasperated, dissatisfied and burdened by the situation, resulting in abuse and subsequently in the respondents experience of decreased QoL. Findings indicate that dependency on others due to physical/ cognitive problems may increase abuse "risk" [53]. On the other hand, the respondents may have expressed frustration and discontentment with their QoL (e.g. poor intimacy), which led to abuse.

Abuse was positively linked to social participation (e.g. increased satisfaction with the opportunity to participate in community activities). This finding seems odd considering that abuse involved, among other acts, being prevented from seeing others. It is possible that although prevented to see others, the respondents nevertheless took part in community activities and valued them highly. Social participation could have functioned as a way to cope with the strains of abuse as the older persons may have been able to express their experiences and received support. Social support has been shown to attenuate the experience of abuse [7,54].

Abuse has been associated with decreased QoL [35,36], but as indicated previously these studies have several limitations (e.g.

${ }^{3-5}$ Daughter. 
Citation: Soares JJF, Sundin Ö, Viitasara E, Melchiorre MG, Stankunas M, et al. (2013) Quality of Life among Persons Aged 60-84 Years in Europe: The Role of Psychological Abuse and Socio-Demographic, Social and Health Factors. J Biosafety Health Educ 1: 101. doi:10.4172/23320893.1000101

Page 5 of 12

\begin{tabular}{|c|c|c|c|c|c|c|}
\hline \multirow[t]{2}{*}{ Independent variables } & \multicolumn{6}{|c|}{ Autonomy Past/present/future activities } \\
\hline & $\boldsymbol{\beta}(\mathbf{S E})^{\prime}$ & $\boldsymbol{\beta}^{m}$ & Cl95 $^{n}$ & $\beta(\mathbf{S E})^{\prime}$ & $\boldsymbol{\beta}^{\mathrm{m}}$ & $\mathrm{Cl} 95^{\mathrm{n}}$ \\
\hline \multicolumn{7}{|l|}{ Country $^{b}$} \\
\hline Greece & $-5.317(1.253)$ & $-0.096^{\star \star \star \star *}$ & $-7.773 /-2.861$ & $-4.855(1.046)$ & $-0.094^{*+* * *}$ & $-6.906 /-2.803$ \\
\hline Italy & $-11.478(1.150)$ & $-0.204^{* \star * *}$ & $-13.733 /-9.223$ & $-7.259(0.961)$ & $-0.138^{+\ldots+\cdots}$ & $-9.144 /-5.374$ \\
\hline Lithuania & $-3.931(1.180)$ & $-0.075^{\star \star \star}$ & $-6.246 /-1.617$ & $-6.257(0.986)$ & $-0.128^{\text {tat* }}$ & $-8.191 /-4.323$ \\
\hline Portugal & $-5.668(1.110)$ & $-0.106^{\star \star * \star}$ & $-7.845 /-3.491$ & $-5.272(0.926)$ & -0.106 & $-7.088 /-3.456$ \\
\hline Spain & $-3.949(1.291)$ & $-0.064^{* *}$ & $-6.481 /-1.417$ & $-6.100(1.081)$ & -0.106 & $-8.219 /-3.980$ \\
\hline Sweden & $-5.566(1.088)$ & $-0.101^{* \star * *}$ & $-7.699 /-3.433$ & $-3.979(0.909)$ & -0.078 & $-5.762 /-2.196$ \\
\hline Germany ${ }^{\circ}$ & & 1 & & & 1 & \\
\hline \multicolumn{7}{|l|}{ Age $^{\mathrm{b}}$} \\
\hline $65-69$ & $0.448(0.812)$ & 0.010 & $-1.145 / 2.041$ & $0.991(0,683)$ & 0.024 & $-0.349 / 2.331$ \\
\hline $70-74$ & $0.252(0.885)$ & 0.005 & $-1.484 / 1.988$ & $0.890(0,744)$ & 0.020 & $-0.568 / 2.349$ \\
\hline $75-79$ & $0.601(0.971)$ & 0.011 & $-1.302 / 2.504$ & $0.736(0.814)$ & 0.015 & $-0.861 / 2.333$ \\
\hline $80-84$ & $-2.665(1.081)$ & $-0.044^{*}$ & $-4.785 /-0.545$ & $-0.023(0.910$ & 0.001 & $-1.806 / 1.761$ \\
\hline $60-64^{\circ}$ & & 1 & & & 1 & \\
\hline \multicolumn{7}{|l|}{ Sex ${ }^{b}$} \\
\hline Male & $-0.430(0.650)$ & -0.011 & $-1.704 / 0.844$ & $-2.116(0.546)$ & 0.032 & $-4.431 / 0.418$ \\
\hline Female $^{c}$ & & 1 & & & 1 & \\
\hline \multicolumn{7}{|l|}{ Migrant background ${ }^{b}$} \\
\hline Yes & $-2.039(1.211)$ & -0.024 & $-4.413 / 0.335$ & $-1.233(1.008)$ & -0.016 & $-3.210 / 0.744$ \\
\hline $\mathrm{No}^{\mathrm{c}}$ & & 1 & & & 1 & \\
\hline \multicolumn{7}{|l|}{ Marital status $^{\mathrm{b}}$} \\
\hline Married/cohabitant & $-2.231(2.058)$ & -0.054 & $-6.266 / 1.804$ & $-0.666(1.717)$ & -0.017 & $-4.033 / 2.700$ \\
\hline Divorced/separated & $0.322(1.469)$ & 0.004 & $-2.557 / 3.202$ & $-2.007(1.235)$ & -0.030 & $-4.428 / 0.415$ \\
\hline Widow/er & $0.560(1.314=$ & 0.011 & $-2.016 / 3.137$ & $0.744(1.103)$ & 0.016 & $-1.419 / 2.906$ \\
\hline Single $^{c}$ & & 1 & & & 1 & \\
\hline Living situation ${ }^{\mathrm{b}}$ & & & & & & \\
\hline Spouse/partner & $-3.399(1.922)$ & -0.087 & $-7.168 / 0.370$ & $-1.463(1.599)$ & -0.040 & $-4.599 / 1.672$ \\
\hline Spouse/partner/other & $-1.932(2.149)$ & -0.037 & $-6.144 / 2.280$ & $-2.866(1.793)$ & -0.058 & $-6.382 / 0.650$ \\
\hline Other ${ }^{f}$ & $-3.928(1.228)$ & -0.039 & $-6.150 / 2.286$ & $-2.178(1.033)$ & -0.034 & $-4.432 / 0.419$ \\
\hline Alone $^{c}$ & & 1 & & & 1 & \\
\hline Housing ${ }^{\mathrm{b}}$ & & & & & & \\
\hline Rent & $-1.707(0.738)$ & -0.026 & $-4.419 / 0.339$ & $-2.907(0.620)$ & -0.033 & $-4.431 / 0.420$ \\
\hline Otherg ${ }^{g}$ & $-3.563(1.609)$ & -0.030 & $-4.543 / 0.343$ & $-1.827(1.354)$ & -0.017 & $-4.482 / 0.828$ \\
\hline Ownc & & 1 & & & 1 & \\
\hline Household size & $-0.543(0.333)$ & -0.033 & $-1.196 / 0.111$ & $0.668(0.280)$ & $0.043^{*}$ & $0.119 / 1.218$ \\
\hline Education $^{\mathrm{b}}$ & & & & & & \\
\hline Low education ${ }^{h}$ & $4.164(2.028)$ & $0.102^{\circ}$ & $0.189 / 8.140$ & $1.483(1.707)$ & 0.039 & $-1.864 / 4.830$ \\
\hline Middle education & $4.958(2.107)$ & $0.124^{\circ}$ & $0.826 / 9.089$ & $1.768(1.774)$ & 0.048 & $-1.710 / 5.246$ \\
\hline High education & $6.067(2.241)$ & $0.128^{* \prime}$ & $1.674 / 10.461$ & $2.505(1.885)$ & 0.057 & $-1.191 / 6.201$ \\
\hline Cannot read/write ${ }^{c}$ & & 1 & & & 1 & \\
\hline Profession $^{b}$ & & & & & & \\
\hline $\begin{array}{l}\text { Blue-collar agricultural/fishery/ } \\
\text { crafts }\end{array}$ & $1.014(0.948)$ & 0.024 & $-0.845 / 2.873$ & $0.219(0.795)$ & 0.006 & $-1.340 / 1.778$ \\
\hline Low white-collar worker & $1.361(0.867)$ & 0.031 & $-0.339 / 3.060$ & $-1.138(0.726)$ & -0.028 & $-2.561 / 0.286$ \\
\hline Armed forces/similar & $1.600(2.588)$ & 0.009 & $-3.474 / 6.675$ & $-0.129(2.179)$ & -0.001 & $-4.400 / 4.143$ \\
\hline Housewives/husbands & $-1.810(1.398)$ & -0.028 & $-4.552 / 0.931$ & $-2.536(1.169)$ & -0.033 & $-0.513 / 3.870$ \\
\hline Middle/high white-collar worker ${ }^{c}$ & & 1 & & & 1 & \\
\hline Financial support ${ }^{\mathrm{b}}$ & & & & & & \\
\hline Work & $1.470(1.327)$ & 0.025 & $-1.133 / 4.072$ & $1.679(1.116)$ & 0.031 & $-0.510 / 3.867$ \\
\hline $\begin{array}{l}\text { Social/sickness/other pension } \\
\text { benefits }\end{array}$ & $-4.672(1.276)$ & $-0.053^{\star \star \star \star *}$ & $-7.175 /-2.170$ & $-2.776(1.071)$ & $-0.035^{\star \star}$ & $-4.876 /-0.675$ \\
\hline Spouse/partner income & $0.262 / 1.190)$ & 0.004 & $-2.070 / 2.595$ & $0.446(0.999)$ & 0.008 & $-1.512 / 2.404$ \\
\hline Other income ${ }^{k}$ & $-1.239(1.829)$ & -0.010 & $-4.825 / 2.347$ & $0.536(1.524)$ & 0.005 & $-2.452 / 3.524$ \\
\hline Work pension $^{c}$ & & 1 & & & 1 & \\
\hline Still wrork ${ }^{\mathbf{b}}$ & & & & & & \\
\hline Yes & $-1.170(1.130)$ & -0.023 & $-3.386 / 1.046$ & $0.263(0.951)$ & 0.006 & $-1.602 / 2.128$ \\
\hline $\mathrm{No}^{\mathrm{c}}$ & & 1 & & & 1 & \\
\hline Financial strain ${ }^{b}$ & & & & & & \\
\hline Yes & $-2.621(0.629)$ & $-0.064^{* * * *}$ & $-3.854 /-1.389$ & $-2.620(0.528)$ & $-0.069^{\star * \star *}$ & $-3.655 /-1.586$ \\
\hline $\mathrm{No}^{\mathrm{c}}$ & & 1 & & & 1 & \\
\hline Smoking $^{b}$ & & & & & & \\
\hline Yes & $0.676(0.859)$ & 0.011 & $-1.009 / 2.361$ & $-1.400(0.725)$ & -0.025 & $-2.821 / 0.021$ \\
\hline $\mathrm{No}^{\mathrm{c}}$ & & 1 & & & 1 & \\
\hline Drinking $^{\text {b }}$ & & & & & & \\
\hline Yes & $2.847(0.650)$ & $0.069 \cdots$ & $1.573 / 4.121$ & $0.412(0.546$ & 0.011 & $-0.660 / 1.483$ \\
\hline $\mathrm{No}^{\mathrm{c}}$ & & 1 & & & 1 & \\
\hline$B M l^{d}$ & $0.111(0.068)$ & 0.023 & $-0.022 / 0.245$ & $0.044(0.057)$ & 0.010 & $-0.068 / 0.155$ \\
\hline Health care use ${ }^{d}$ & $0.104(0.102)$ & 0.015 & $-0.096 / 0.303$ & $0,071(0.086)$ & 0.011 & $-0.098 / 0.239$ \\
\hline Physical diseases $^{d}$ & $0.396(0.223)$ & 0.029 & $-0.041 / 0.833$ & $-0.126(0.188)$ & -0.010 & $-0.494 / 0.242$ \\
\hline Anxiety symptoms ${ }^{d}$ & $-0.286(-0.286)$ & $-0.058^{\star *}$ & $-0.466 /-0.105$ & $-0.538(0.077)$ & $-0.118^{* * \star *}$ & $-0.689 /-0.387$ \\
\hline Depressive symptoms ${ }^{d}$ & $-1.470(0.099)$ & $-0.302^{\star \star \star \star}$ & $-1.664 /-1.276$ & $-1.620(0.083)$ & $-0.357^{\star \star \star \star}$ & $-1.782 /-1.458$ \\
\hline Somatic symptoms ${ }^{\mathrm{d}}$ & $-0.138(0.026)$ & $-0.103^{\star \star \star \star}$ & $-0.188 /-0.087$ & $-0.061(0.022)$ & $-0.049^{\star \star}$ & $-0.103 /-0.018$ \\
\hline Social support ${ }^{d}$ & $0.223(0.021)$ & $0.164^{\star \star \star \star}$ & $0.181 / 0.265$ & $0.311(0.018)$ & $0.247^{\star \star \star \star}$ & $0.276 / 0.347$ \\
\hline Psychological abuse $^{\mathrm{b}}$ & & & & & & \\
\hline Yes & $-2.482(0.699)$ & $-0.051^{\star \star \star \star *}$ & $-3.852 /-1.113$ & $-1.643(0.586)$ & $-0.036^{\star *}$ & $-2.792 /-0.494$ \\
\hline $\mathrm{No}^{\mathrm{c}}$ & & 1 & & & 1 & \\
\hline $\mathrm{R}^{2}$ & $32.8 \%$ & & & $44.8 \%$ & & \\
\hline
\end{tabular}

a=WHOQOL-OLD, sub-scales; ${ }^{\mathrm{b}}=$ categorical variables; ${ }^{\mathrm{c}}=$ comparison variable; ${ }^{\mathrm{d}}=$ continuous variables; ${ }^{\mathrm{e}}=\mathrm{e} . \mathrm{g}$. daughter; ${ }^{\mathrm{f}}=$ without spouse/partner, but other e.g. daughter

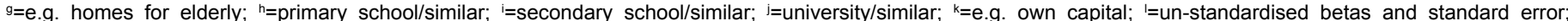
$\mathrm{m}=$ standardized betas; $\mathrm{m}=$ lower/upper bound; VIF's for autonomy ranged from 1.085-8.552; VIF's for past/present/future activities range from 1.067-7.079; * $\mathrm{p}<0.05$; ${ }^{* *} p<0.01 ;{ }^{* * *} p<0.001 ;{ }^{* * * *} p<0.0001$.

Table 2: Correlates of quality of life by domain (multiple linear regression analyses). 
Citation: Soares JJF, Sundin Ö, Viitasara E, Melchiorre MG, Stankunas M, et al. (2013) Quality of Life among Persons Aged 60-84 Years in Europe: The Role of Psychological Abuse and Socio-Demographic, Social and Health Factors. J Biosafety Health Educ 1: 101. doi:10.4172/23320893.1000101

Page 6 of 12

\begin{tabular}{|c|c|c|c|c|c|c|}
\hline \multirow[t]{2}{*}{ Independent variables } & \multicolumn{2}{|c|}{ Social participation } & \multicolumn{4}{|c|}{ Intimacy } \\
\hline & $\boldsymbol{\beta}(\mathbf{S E})^{\prime}$ & $\boldsymbol{\beta}^{\mathrm{m}}$ & $\mathrm{Cl}^{2} 5^{\mathrm{n}}$ & $\boldsymbol{\beta}(\mathbf{S E})^{\prime}$ & $\boldsymbol{\beta}^{\mathrm{m}}$ & $\mathrm{Cl}^{2} 5^{\mathrm{n}}$ \\
\hline \multicolumn{7}{|l|}{ Country $^{b}$} \\
\hline Greece & $-2.976(1.153)$ & $-0.054^{\star \star}$ & $5.238 /-0.715$ & $-9.748(1.681)$ & $-0.117^{* \star \star \star}$ & $-13.044 /-6.453$ \\
\hline Italy & $-5.424(1.061)$ & $-0.096^{* * * *}$ & $-7.504 /-3.344$ & $-9.736(1.543)$ & $-0.115^{* * * *}$ & $-12.761 /-6.712$ \\
\hline Lithuania & $-2.795(1.086)$ & $-0.054^{\star *}$ & $-4.925 /-0.665$ & $-32.025(1.585)$ & $-0.407^{* \star \star *}$ & $-35.133 /-28.917$ \\
\hline Portugal & $-2.722(1.020)$ & $-0.051^{\star *}$ & $-4.722 /-0.723$ & $-8.947(1.497)$ & $-0.109^{\star * * *}$ & $-11.882 /-6.012$ \\
\hline Spain & $-3.285(1.190)$ & $-0.054^{\star \star}$ & $-5.617 /-0.952$ & $-11.269(1.737)$ & $-0.122^{\star \star \star \star}$ & $-14.675 /-7.863$ \\
\hline Sweden & $-5.979(0.999)$ & $-0.109^{* * * *}$ & $-7.938 /-4.021$ & $-13.957(1.462)$ & $-0.169^{\star \star \star \star}$ & $-16.823 /-11.090$ \\
\hline Germany $^{\mathrm{c}}$ & & 1 & & & 1 & \\
\hline \multicolumn{7}{|l|}{$\mathrm{Age}^{\mathrm{b}}$} \\
\hline $65-69$ & $0.771(0.753)$ & 0.017 & $-0.705 / 2.248$ & $-0.150(1.099)$ & -0.002 & $-2.305 / 2.006$ \\
\hline $70-74$ & $0.739(0.823)$ & 0.016 & $-0.874 / 2.352$ & $-2.212(1.196)$ & -0.031 & $-4.556 / 0.132$ \\
\hline $75-79$ & $0.302(0.898)$ & 0.006 & $-1.460 / 2.063$ & $0.697(1.311)$ & 0.009 & $-1.873 / 3.267$ \\
\hline $80-84$ & $-3.357(1.002)$ & $-0.056^{\star * *}$ & $-5.322 /-1.391$ & $-1.708(1.465)$ & -0.019 & $-4.581 / 1.165$ \\
\hline $60-64^{c}$ & & 1 & & & 1 & \\
\hline \multicolumn{7}{|l|}{ Sex ${ }^{b}$} \\
\hline Male & $-3.454(0.601)$ & $-0.088^{\star \star * \star}$ & $-4.633 /-2.275$ & $1.030(0.878)$ & 0.017 & $-0.691 / 2.750$ \\
\hline Female $^{c}$ & & 1 & & & 1 & \\
\hline \multicolumn{7}{|l|}{ Migrant background ${ }^{b}$} \\
\hline Yes & $-2.138(1.116)$ & -0.025 & $-4.325 / 0.049$ & $-0.474(1.641)$ & -0.004 & $-3.691 / 2.743$ \\
\hline $\mathrm{No}^{\mathrm{C}}$ & & 1 & & & 1 & \\
\hline Marital status ${ }^{b}$ & & & & & & \\
\hline Married/cohabitant & $-3.601(1.890)$ & -0.087 & $-7.308 / 0.105$ & $7.299(2.772)$ & $0.117^{* * *}$ & $1.864 / 12.734$ \\
\hline Divorced/separated & $-2.527(1.358)$ & -0.035 & $-5.189 / 0.136$ & 1.262(1.998) & 0.011 & $-2.656 / 5.180$ \\
\hline Widow/er & $-1.625(1.213)$ & -0.033 & $-4.003 / 0.754$ & $0.209(1.785$ & 0.003 & $-3.291 / 3.709$ \\
\hline Single ${ }^{c}$ & & 1 & & & 1 & \\
\hline Living situation ${ }^{b}$ & & & & & & \\
\hline Spouse/partner & $-0.414(1.761)$ & -0.011 & $-3.868 / 3.040$ & $10.620(2.582)$ & $0.180^{\star \star \star \star}$ & $5.557 / 15.683$ \\
\hline Spouse/partner/other ${ }^{\mathrm{e}}$ & $-1.080(1.975)$ & -0.020 & $-4.953 / 2.793$ & $9.544(2.892)$ & $0.120^{* \star *}$ & $3.874 / 15.214$ \\
\hline Otherf & $-3.118(1.141)$ & -0.037 & $-5.191 / 0.139$ & $4.133(1.682)$ & $0.042^{* *}$ & $0.836 / 7.430$ \\
\hline Alone $^{c}$ & & 1 & & & 1 & \\
\hline Housing $^{b}$ & & & & & & \\
\hline Rent & $-0.656(0.682)$ & -0.014 & $-1.994 / 0.681$ & $-0.223(0.999)$ & -0.003 & $-2.182 / 1.736$ \\
\hline Other ${ }^{g}$ & $-0.905(1.493)$ & -0.008 & $-3.831 / 2.021$ & $-4.075(2.218)$ & -0.024 & $-8.424 / 0.274$ \\
\hline Ownc & & 1 & & & 1 & \\
\hline Household size & $0.090(0.309)$ & 0.005 & $-0.515 / 0.696$ & $0.973(0.451)$ & $0.039^{*}$ & $0.089 / 1.857$ \\
\hline Education $^{\mathrm{b}}$ & & & & & & \\
\hline Low Education $^{\mathrm{h}}$ & $0.302(1.881)$ & 0.007 & $-3.386 / 3.990$ & $-0.375(2.782)$ & -0.006 & $-5.829 / 5.080$ \\
\hline Middle Education & $0.242(1.955)$ & 0.006 & $-3.590 / 4.075$ & $1.038(2.889)$ & 0.017 & $-4.625 / 6.702$ \\
\hline High Education & $0.504(2.078)$ & 0.011 & $-3.570 / 4.579$ & $1.483(3.063)$ & 0.021 & $-4.523 / 7.488$ \\
\hline Cannot read/write ${ }^{c}$ & & 1 & & & 1 & \\
\hline Profession $^{\mathrm{b}}$ & & & & & & \\
\hline Blue-collar/agricultural/fishery/crafts & $0.217(0.878)$ & 0.005 & $-1.504 / 1.939$ & $-0.549(1.281)$ & -0.009 & $-3.062 / 1.963$ \\
\hline Low white-collar worker & $-0.224(0.800)$ & -0.005 & $-1.794 / 1.345$ & $-0.716(1.169)$ & -0.011 & $-3.007 / 1.576$ \\
\hline Armed forces/similar & $2.936(2.427)$ & 0.016 & $-1.822 / 7.694$ & $3.700(3.499)$ & 0.013 & $-3.159 / 10.560$ \\
\hline Housewives/husbands & $-1.229(1.293)$ & -0.019 & $-3.765 / 1.306$ & $-0.333(1.877)$ & -0.003 & $-4.013 / 3.347$ \\
\hline Middle/high white-collar worker ${ }^{c}$ & & 1 & & & 1 & \\
\hline Financial support ${ }^{\mathrm{b}}$ & & & & & & \\
\hline Work & $-1.161(1.232)$ & -0.020 & $-3.577 / 1.255$ & $-0.052(1.810)$ & 0.001 & $-3.600 / 3.496$ \\
\hline Social/sickness/other pension benefits & $-5.090(1.179)$ & $-0.058^{*+* t *}$ & $-7.401 /-2.779$ & $2.297(1.740)$ & 0.017 & $-1.115 / 5.708$ \\
\hline Spouse/partner income & $0.017(1.100)$ & 0.001 & $-2.140 / 2.173$ & $3.885(1.612)$ & $0.041^{\star}$ & $0.725 / 7.046$ \\
\hline Other income ${ }^{k}$ & $0.969(1.681)$ & 0.008 & $-2.326 / 4.264$ & $1.234(2.461)$ & 0.006 & $-3.592 / 6.060$ \\
\hline Work pension $^{c}$ & & 1 & & & 1 & \\
\hline Still work ${ }^{b}$ & & & & & & \\
\hline Yes & $3.634(1.052)$ & $0.071^{* * *}$ & $1.571 / 5.696$ & $2.516(1.547)$ & 0.033 & $-0.517 / 5.549$ \\
\hline $\mathrm{No}^{\mathrm{c}}$ & & 1 & & & & 1 \\
\hline & & & & & & \\
\hline Financial strain ${ }^{b}$ & & & & & & \\
\hline Yes & $-1.044(0.582)$ & -0.026 & $-2.186 / 0.098$ & $2.760(0.848)$ & $0.045^{\star * *}$ & $1.097 / 4.423$ \\
\hline $\mathrm{No}^{\mathrm{c}}$ & & 1 & & & 1 & \\
\hline Smoking ${ }^{b}$ & & & & & & \\
\hline Yes & $-1.601(0.798)$ & $-0.027^{*}$ & $-3.166 /-0.037$ & $-0.758(1.159)$ & -0.008 & $-3.030 / 1.515$ \\
\hline $\mathrm{No}^{\mathrm{c}}$ & & 1 & & & 1 & \\
\hline Drinking ${ }^{b}$ & & & & & & \\
\hline Yes & $1.504(0.603)$ & $0.037^{\star *}$ & $0.321 / 2.686$ & $0.489(0.881)$ & 0.008 & $-1.238 / 2.217$ \\
\hline $\mathrm{No}^{\mathrm{c}}$ & & 1 & & & 1 & \\
\hline $\mathrm{BMI}^{\mathrm{d}}$ & $-0.101(0.063)$ & -0.021 & $-0.224 / 0.022$ & $-0.102(0.092)$ & -0.014 & $-0.282 / 0.078$ \\
\hline Health care use $\mathrm{d}^{\mathrm{d}}$ & $-0.006(0.094)$ & -0.001 & $-0.191 / 0.179$ & $0.429(0.138)$ & $0.042^{* * * *}$ & $0.159 / 0.700$ \\
\hline Physical diseases $^{d}$ & $-0.124(0.207)$ & -0.009 & $-0.531 / 0.282$ & $0.431(0.302)$ & 0.021 & $-0.162 / 1.024$ \\
\hline Anxiety symptoms ${ }^{d}$ & $-0.217(0.085)$ & $-0.044^{* *}$ & $-0.384 /-0.050$ & $-0.471(0.124)$ & $-0.064^{\star * \star *}$ & $-0.714 /-0.228$ \\
\hline Depressive symptoms $^{d}$ & $-2.044(0.092)$ & $-0.420^{* * * *}$ & $-2.223 /-1.864$ & $-1.358(0.134)$ & $-0.185^{\star * * *}$ & $-1.620 /-1.096$ \\
\hline Somatic symptoms ${ }^{d}$ & $-0.138(0.024)$ & $-0.104^{* * * *}$ & $-0.185 /-0.091$ & $-0.015(0.035)$ & -0.007 & $-0.083 / 0.053$ \\
\hline Social support ${ }^{d}$ & $0.269(0.020)$ & $0.200^{\star \star \star *}$ & $0.230 / 0.308$ & $0.530(0.029)$ & $0.258^{* \star * *}$ & $0.473 / 0.586$ \\
\hline Psychological abuse $^{b}$ & & & & & & \\
\hline Yes & $1.517(0.647)$ & $0.31^{* *}$ & $0.249 / 2.784$ & $-6.861(0.945)$ & $-0.094^{* * * *}$ & $-8.714 /-5.007$ \\
\hline $\mathrm{No}^{\mathrm{C}}$ & & 1 & & & 1 & \\
\hline $\mathrm{R}^{2}$ & $41.5 \%$ & & & $45.6 \%$ & & \\
\hline
\end{tabular}

${ }^{a}=$ WHOQOL-OLD, sub-scales; ${ }^{b}=$ categorical variables; ${ }^{c}=$ comparison variable; ${ }^{d}=$ continuous variables; ${ }^{e}=e . g$. daughter; ${ }^{f}=$ without spouse/partner, but other e.g daughter; ${ }^{\mathrm{g}}=\mathrm{e} . \mathrm{g}$. homes for elderly; ${ }^{\mathrm{h}}=$ primary school/similar; ${ }^{\mathrm{i}}=$ secondary school/similar; ${ }^{\mathrm{j}}=$ university/similar; ${ }^{k}=\mathrm{e} . \mathrm{g}$. own capital; $=$ =un-standardised betas and standard error; ${ }^{m}=$ standardised betas; ${ }^{m}=$ lower/upper bound; VIF's of social participation ranged from 1.066-9.596; VIF's of intimacy ranged from $1.066-8.943$; $^{*} p<0.05$; ${ }^{* *} p<0.01$; ${ }^{\star * *} p<0.001 ;{ }^{* * * *} p<0.0001$.

Table 3: Correlates of quality of life by domain (multiple linear regression analyses). 
methodological). In any case, our study may be the first to demonstrate a clear relationship between psychological abuse and decreased QoL, although mainly regarding autonomy, intimacy and past/present/future activities.

\section{QoL and Country}

All QoL domains (autonomy, intimacy, past/present/future activities, social participation) were negatively related to being from any other country in contrast to Germany (reference country). This in line with a study across 21 countries (e.g. Germany), i.e. older persons from developing countries scored lower on these domains than those from medium and high-development countries [55]. However, it is hazardous to compare results as only four of our countries were included (Germany, Lithuania, Spain, Sweden) and none of these were developing countries.

Further, other data show that individuals from our countries (except Sweden) contrasted to those from Germany experience less autonomy, with some of the underlying reasons being lower levels of social services and incomes [24-27]. The discrepancy regarding Sweden may pertain to that the financial situation of older persons in this country, particularly the oldest, has deteriorated during the past years (e.g. low incomes) and this could have had a negative effect on their autonomy [56]. Additional findings show that autonomy depends on various factors such as disease, financial resources and social support [26,57-61].

As to intimacy, available findings tend to involves elected groups such as frail older persons and the bulk of studies focus on the sexual expression of intimacy and emphasize its importance for well-being [62-66]. Our findings are unlikely to reflect that the respondents from the other included countries compared to those of Germany differed in their living situation (who lived with the respondent) and were more often singles, divorced/separated or widowers in view that there were no major differences in total percentages. It is possible for instance that the respondents from the other included countries compared to those from Germany experienced a less "tolerant" environment to give/ receive intimacy, and therefore the lower scores, but this issue was not directly addressed.

Regarding past/present/future activities, it is likely that the expression of achievement values among older persons depends on factors such as life satisfaction, self-esteem and individual/societal economic development. In line with this, data indicate that older persons in most of the included countries experienced a lower life satisfaction contrasted to those from Germany [24,27] due to various reasons such as economic difficulties. Poverty levels among older persons in Germany are lower than in most of our included countries $[67,68]$. Self-esteem declines in older age $[69,70]$, but the decline may have been less evident among respondents in Germany. Thus, the respondents satisfaction with opportunities in life and how satisfied they were with what they achieved in life may have been influenced by the above mentioned factors.

As to social participation, it is likely that poverty levels $[67,68]$ were an obstacle for participation in social activities, at least in some countries such as Portugal, and data show indeed that low income levels are associated with decreased QoL [24,27]. Decreased social participation could also be an indirect measure of loneliness. Older persons from Southern European countries feel lonelier than those from Germany pertaining to factors such as economic deprivation, although our results concerning Sweden and Lithuania seem at odds
[71,72]. Poverty, loneliness or both could thus be an obstacle for social participation and therefore the dissatisfaction.

\section{QoL, Demographics/socio-Economics and Life-Style}

Age 80-84 years was negatively associated with autonomy and social participation. This at odds with results from Norway, i.e. no differences in these domains between persons aged below 75 years and those aged 75 years and over [73]. Sample differences, for instance, may explain the discrepancy. In general, older cohorts compared to younger have worse health, depend more on others, are more isolated and have lower incomes, and these factors negatively affect their well-being and QoL $[67,68,74-82]$. Thus, such circumstances may have hindered our respondents to do the things they like to do and to take part in activities as desired.

Male gender was negatively associated with social participation. Thisis in line with a Norwegian study among older adults, i.e. men scored lower on social participation than women [73]. Our findings seem to reflect that older men contrasted to older women have less extensive social ties and participate less in social activities $[83,84]$.

Being married/cohabitant, living with spouse/partner/other ${ }^{5}$ and in large households were positively associated with intimacy. This indicates that spouses/partners and significant other are vital for the well-being of older persons as for instance givers of affection. Family and significant other also provide companionship and support when health declines and the older person need help. Our findings seem to bean indirect indicator that some of our respondents lived in harmonious relationships. For example, harmonious marriages positively affect for instance the psychological well-being of individuals, including older persons [85-91]. Living in large households suggests that the older persons had multi-levels of support and opportunities to receive/give love, and indeed living in extended families and/or receiving inter generational support has been shown to provide benefits for older persons, particularly in relation to health $[92,93]$ although the quality of the relationships in the family may be more important than the number of persons in it [94]. Thus, living in large households may also be beneficial for intimacy.

All types of education compared to no education were positively associated with autonomy. Contrary to our findings, a recent Brazilian study with older adults reported no major impact of education on autonomy [95]. Our findings seem also at odds with data showing that individuals, including older persons, with high educational attainment experience greater QoL than those with lower educational attainment. However, we confirmed that individuals with the highest educational attainment report greater QoL than those without any educational attainment and the opposite [96-98]. Differences, for instance, in the definition of educational levels may account for the discrepancy between our findings and those of others.

Financial support based on social/sickness benefits/other pension benefits was negatively associated with autonomy, past/present/future activities and social participation. Being supported by benefits (e.g. social) indicates financial difficulties and thus in contrast to higher social groups those who are on benefits may be less happy and in control of their situation [99]. Further, there is a link between income and health which affects QOL, showing that within countries, poorer health is associated with lower income [100,101]. Low income levels seem also to lead to decreased QoL [24,27]. On the other hand, the above mentioned association could reflect that people with poor health, which affects QOL, are more likely to be on special types of benefits 
Citation: Soares JJF, Sundin Ö, Viitasara E, Melchiorre MG, Stankunas M, et al. (2013) Quality of Life among Persons Aged 60-84 Years in Europe: The Role of Psychological Abuse and Socio-Demographic, Social and Health Factors. J Biosafety Health Educ 1: 101. doi:10.4172/23320893.1000101

Page 8 of 12

(e.g. social) usually a sign of economic problems [102-104]. Thus, disease, financial problems or both may have hindered respondents to make own decisions freely and to participate in activities to the extent desired, and thus experienced that there wasn't much to look forward to.

Financial strain was negatively associated with autonomy, intimacy and past/present/future activities, illustrating further the importance of finances for the older person's well-being. Income levels decline in older age and there is a close relationship between poverty rates and older ages $[67,68]$. The rates of poverty among older persons are greater than in the population as a whole in some Southern/Eastern European countries, but also in Nordic countries incertain aspects such as low net real assets, and during the past years the financial situation in Europe has deteriorated with increases in living costs and cuts or stagnation of benefits/services $[56,67,68,105-107]$. These circumstances are likely to have led to the experience of financial strain, and, consequently to decreased QOL in various domains. Financial strain may have hindered the respondents to make own decisions about different aspects of their lives and to participate in activities. Financial strain may have also led to disagreements between respondents and those close to them, which would be an obstacle to give and receive love. Studies have shown indeed an association between financial strain/problems/income inequality, poor mental/physical health and decreased QOL among different groups, including older persons [108-112].

An additional finding on the importance of economy in QoL was the positive association between still working and social participation. Employment tends to provide the status, self-esteem and financial resources, which facilitates social relationships, social connections or participation in social activities, and thus the high scores in social participation. However, data on the relation between still working (paid work) and social participation are however ambiguous, with findings indicating that work impacts positively on social participation [113] and others not [114].

Being financially supported by the spouses/partners income was positively associated with intimacy. Financial dependency on a spouse/ partner concerning one's living situation is indicative of little decision latitude for the dependent person and greater influence in how the resources are spent and who spends them for the financial contributor [115-117]. A longitudinal analysis of dual-earner couples showed that the husband's financial dependence was linked with lower levels of perceived marital quality among husbands [118], but cross-sectional data found no connection between a husband's economic dependence and his reports of marital satisfaction [119]. Further, recent findings suggest an association between marital dissatisfaction and poorer physical health over time for both men and women, particularly among older persons [120], and being the secondary earner seems to have negative effects on the health of highest-income men [121]. Thus, one could expect financial dependency to be detrimental to intimacy because of marital discord, negative effects on health or both. We found the opposite, i.e. financial dependency was related to increased satisfaction with intimacy. A plausible explanation is that at the age of the respondents issues regarding, for instance, who is the bread winner and who has the final say played little role. The couples were likely to share financial intimacy and this had a positive influence on intimacy.

Alcohol use was positively associated with social participation and autonomy. Slightly over $64 \%$ of our respondents used alcohol and $82.7 \%$ drank 1-2 drinks a day, indicating that they were moderate drinkers ${ }^{6}$. Of the alcohol users, $68.2 \%$ used alcohol in conjunction with social activities, e.g. meeting friends. ${ }^{7}$ Moderate alcohol use has been associated with beneficial effects on mortality risk, health and QoL among older persons [122-125]. Thus, the positive relation between alcohol use and social participation may be a reflection of this. As to the relation between autonomy and alcohol use, one could hypothesize that alcohol use gave the respondents a sense that they could make their own decisions.

\section{QoL and health}

Not surprisingly, depressive and anxiety symptoms were negatively associated with all QoL domains, and somatic symptoms with autonomy, past/present/future activities and social participation. Depression, anxiety and somatic symptoms have been associated with decreased QoL among various types of elder samples and in different settings/ countries [28-32,76], and in a recent study among older persons it was found that psychological well-being predicted QoL [126]. Thus, our findings seem to confirm those of a plethora of studies. The mechanisms underlying the connection between these conditions and decreased QoL are likely to be complex. For example, depressed persons may have reduced interest or pleasure in all activities, or almost all; diminished ability to make own decisions; and are unlikely to engage and/or find satisfaction, or at least seldom, in such events as participation in daily activities and give intimacy. Hence, it may be foreseeable that they report a decreased QoL. On the other hand, a reverse pattern could be possible in some cases. For instance, persistent refusal of intimacy and lack of appreciation over achievements could over time have led to depressive, anxiety or somatic symptoms.

\section{QoL and social support}

High scores in social support were positively related to all QoL domains. Social support (e.g. having help from friends) ${ }^{8}$ and social engagement has a positive influence on health, QoL and life satisfaction and the opposite regarding low social support/social isolation [33,34,127-130]. Thus, our results seem in accord with previous observations. The mechanisms underlying the connection between social support and the QoL domains were not addressed in our study. Nevertheless, it is possible for example that social support functioned as a "mechanism" buffering the negative effects of stress and enhancing personal coping abilities such as self-esteem and self-efficacy [131], which would positively affect the older persons perception of various components of $\mathrm{QoL}$ as receivers and givers.

\section{Limitations}

This study has limitations. First, the cross-sectional nature of the data did not allowed to firmly conclude about causality. Second, the respondents may not have been representative for rural samples and other countries in Europe or elsewhere (e.g. USA) as they were recruited in urban centers from only seven specific European countries. Thus, the generalizability of our findings cannot be guaranteed. Third, the non-

${ }^{6}$ One drink represents one unit and is equivalent to 10 grams of alcohol

${ }^{7}$ Details on alcohol use patterns and activities are not shown here.

${ }_{8,9}$ In some cases the studies include also young persons. 
Citation: Soares JJF, Sundin Ö, Viitasara E, Melchiorre MG, Stankunas M, et al. (2013) Quality of Life among Persons Aged 60-84 Years in Europe: The Role of Psychological Abuse and Socio-Demographic, Social and Health Factors. J Biosafety Health Educ 1: 101. doi:10.4172/23320893.1000101

Page 9 of 12

use of objective measures to corroborate the respondents' subjective assessments of their situation affects the accuracy. For example, the presence of somatic symptoms (e.g. pain) was not objectively confirmed. On the other hand, the used instrument (GBB) has good psychometric properties and is sensitive to age [e.g. 43]. Fourth, GBB, Multidimensional Scale of Perceived Social Support and health items needed to be translated into some of the included country's languages, back-translated and culturally adapted. Although this was done with great caution and precision, errors could have occurred raising questions about the validity of what was measured. Fifth, the high non-response rate could have led to the "selection" of women/men with characteristics differing from those of women/men in general. For instance, we may have an over-representation of men who were psychologically abused [7]. However, there were no major divergences (age and gender) between responders and non-responders nor did they differ from the population in each participating state [37]. Sixth, the burden of the perpetrators such as spouses/cohabitants and offspring was not assessed and thus we cannot conclude on whether it influenced their abusive behaviors. Seventh, sleep disturbances are common among older persons [132,133] and may lead to deteriorated QoL $[134,135]$, but this issue was not directly addressed here precluding any conclusion about its influence on QoL. In spite of the limitations, the strength of this study lies in its careful methodology, large sample and multi-country approach. It also provides an overview and opportunity to compare older persons from cities in seven European countries with respect to the impact of psychological abuse on QoL by domain considering other factors such as depression and social support.

\section{Conclusions}

Several of the QoL domains were negatively affected by psychological abuse, but other factors such as depressive and anxiety symptoms and country of origin also impacted negatively on QoL. Social support, for example, had a positive effect on the QoL dimensions. Interventions to improve the QoL of older persons should consider these factors, not the least the role of psychological abuse, social support and mental health. Overall, our results seem to have shed further light on the experience of QoL in relation to psychological abuse and other factors such as mental health and social support in older persons; may be useful for changing advocacy and policies regarding older person's experience of QoL, but also for changing public perceptions of it; pointed to the importance of psychological abuse, mental health and social support for the experience of QoL; may be useful for the development of interventions to improve QoL, but also for the development of prevention/treatment interventions to deal with psychological abuse and to decrease mental health and social isolation; and may serve as stimulation for further research across cultures and considering the relationship between various types of elder abuse, QoL, mental health and social support. Notwithstanding, our findings reveal that the QoL of older persons is influenced by many factors, which could not be firmly disentangle. More research, in particular longitudinal, is therefore necessary to conclude about causality.

\section{Authors' contributions}

Joaquim J.F. Soares has made significant contributions to the conception, design, data collection, analyses and interpretation of data. He was also involved in drafting the manuscript and revising it critically. EV and ÖS were involved in the analyses and interpretation of data, and in drafting the manuscript and revising it. MGM, MS, JL, FT-G, HB and EI-K were involved in the gathering, analyses and interpretation of data, and in drafting the manuscript and revising it.

\section{References}

1. United Nations (2010) World population ageing 2009. Department of Economic and Social Affairs, Population Division. New York, United Nations.

2. WHO (2002) The world report on violence and health. World Health Organization, Geneva.

3. WHO (2011) European report on preventing elder maltreatment. World Health Organization, Regional Office for Europe, Copenhagen.

4. Cooper C, Selwood A, Livingston G (2008) The prevalence of elder abuse and neglect: a systematic review. Age Ageing 37: 151-160.

5. Acierno R, Hernandez MA, Amstadter AB, Resnick HS, Steve K, et al. (2010) Prevalence and correlates of emotional, physical, sexual, and financial abuse and potential neglect in the United States: the National Elder Mistreatment Study. Am J Public Health 100: 292-297.

6. Lowenstein A, Eisikovits Z, Band-Winterstein T, Enosh G (2009) Is elder abuse and neglect a social phenomenon? Data from the First National Prevalence Survey in Israel. J Elder Abuse Negl 21: 253-277.

7. Macassa G, Viitasara E, Sundin Ö, Barros H, Gonzales FT, et al (2013) Psychological abuse among older persons in Europe: A cross-sectional study. J Aggress Confl Peace Res 5:16-34.

8. AbathMde B, Leal MC, MeloFilho DA, Marques AP (2010) Physical abuse of older people reported at the Institute of Forensic Medicine in Recife, Pernambuco State, Brazil. Cad SaudePublica 26: 1797-1806.

9. Bonnie R, Wallace R (2003) Risk Factors for Elder Mistreatment. In Elder mistreatment: Abuse, neglect, and exploitation in an aging America. Edited by Bonnie R, Wallace R. Washington, DC: The National Academies Press.

10. Dong X, Simon MA (2008) Is greater social support a protective factor against elder mistreatment? Gerontology 54: 381-388.

11. Dong X, Simon M, Mendes de Leon C, Fulmer T, Beck T, et al. (2009) Elder self-neglect and abuse and mortality risk in a community-dwelling population. JAMA 302: 517-526.

12. Dong XQ, Simon MA, Beck TT, Farran C, McCann JJ, et al. (2011) Elder abuse and mortality: the role of psychological and social wellbeing. Gerontology 57: 549-558.

13. Fulmer T, Paveza G, VandeWeerd C, Fairchild S, Guadagno L, et al. (2005) Dyadic vulnerability and risk profiling for elder neglect. Gerontologist 45: 525 534.

14. Lachs MS, Williams CS, O'Brien S, Pillemer KA, Charlson ME (1998) The mortality of elder mistreatment. JAMA 280: 428-432.

15. Lee M, Kolomer SR (2005) Caregiver burden, dementia, and elder abuse in South Korea. J Elder Abuse Negl 17: 61-74.

16. Shugarman LR, Fries BE, Wolf RS, Morris JN (2003) Identifying older people at risk of abuse during routine screening practices. J Am Geriatr Soc 51: 24-31.

17. Cambridge P, Beadle-Brown J, Milne A, Mansell J, Whelton R (2006) Exploring the incidence, risk factors, nature and monitoring of adultprotection alerts. Canterbury: Tizard Centre.

18. McCallum J, Matiasz S, Graycar A (1990) Abuse of the elderly at home: The range of theproblem. National Centre for Epidemiology and Population Health. Canberra.

19. Abbey L (2009) Elder abuse and neglect: when home is not safe. Clin Geriatr Med 25: 47-60, vi.

20. Kim OS, Yang KM, Kim KH (2005) [Dependency, abuse, and depression by gender in widowed elderly]. Taehan Kanho Hakhoe Chi 35: 336-343.

21. Wolf RS (1997) Elder abuse and neglect: causes and consequences. J Geriatr Psychiatry 30: 153-174.

22. Wu L, Chen H, Hu Y, Xiang H, Yu X, et al. (2012) Prevalence and associated factors of elder mistreatment in a rural community in People's Republic of China: a cross-sectional study. PLoS One 7: e33857.

23. Swagerty DL Jr, Takahashi PY, Evans JM (1999) Elder mistreatment. Am Fam Physician 59: 2804-2808.

24. Anderson R, Mikuliç B Vermeylen G, Lyly-Yrjanainen M, Zigante V (2009) Second European quality of life survey: Overview. European foundation for the improvement of living and working conditions. 
Citation: Soares JJF, Sundin Ö, Viitasara E, Melchiorre MG, Stankunas M, et al. (2013) Quality of Life among Persons Aged 60-84 Years in Europe: The Role of Psychological Abuse and Socio-Demographic, Social and Health Factors. J Biosafety Health Educ 1: 101. doi:10.4172/23320893.1000101

Page 10 of 12

25. KnesebeckOvd, Wahrendorf M, Hyde M, Siegrist J (2007) Socio-economic position and quality of life among older people in 10 European countries: results of the SHARE study. Ageing Soc 27: 269-284.

26. Walker A (2005) Quality of life in old age in Europe. In Growing older in Europe. Editor: Walker A. Suffolk: Open University Press.

27. Watson D, Pichler F, Wallace C (2010) Second European quality of life survey subjective well-being in Europe. European Foundation for the Improvement of Living and Working Conditions.

28. Baumeister H, Hutter N, Bengel J, Härter M (2011) Quality of life in medically ill persons with comorbid mental disorders: a systematic review and metaanalysis. Psychother Psychosom 80: 275-286.

29. Brenes GA (2007) Anxiety, depression, and quality of life in primary care patients. Prim Care Companion J Clin Psychiatry 9: 437-443.

30. Gallegos-Carrillo K, Mudgal J, Sánchez-García S, Wagner FA, Gallo JJ, et al. (2009) Social networks and health-related quality of life: a population based study among older adults. Salud Publica Mex 51: 6-13.

31. Halvorsrud L, Kalfoss M, Diseth $\AA$, Kirkevold M (2012) Quality of life in olde Norwegian adults living at home: a cross-sectional survey. J Res Nurs 17 : 12-29.

32. Porensky EK, Dew MA, Karp JF, Skidmore E, Rollman BL, et al. (2009) The burden of late-life generalized anxiety disorder: effects on disability, healthrelated quality of life, and healthcare utilization. Am J Geriatr Psychiatry 17 473-482

33. Hawton A, Green C, Dickens AP, Richards SH, Taylor RS, et al. (2011) The impact of social isolation on the health status and health-related quality of life of older people. Qual Life Res 20: 57-67.

34. Fernandez-Ballesteros R (2002) Social support and quality of life among older people in Spain. J Soc Issues 58: 645-659.

35. Dong X, Simon MA, Gorbien M (2007) Elder abuse and neglect in an urban chinese population. J Elder Abuse Negl 19: 79-96.

36. O' Keeffe M (2007) UK study of abuse and neglect of older people: Prevalencesurvey report. London: Department of health.

37. Lindert J, Luna J, Torres-Gonzalez F, Barros H, loannidi-Kapolou E, et al (2012) Study design, sampling and assessment methods of the European study 'abuse of the elderly in the European region'. Eur J Public Health 22 662-666

38. Power M, Quinn K, Schmidt S (2005) Development of the WHOQOL-old module. Qual Life Res 14: 2197-2214.

39. The WHOQOL Group (1994) Development of the WHOQOL: Rationale and current status. Int J Ment Health 23: 24-56.

40. (1995) The World Health Organization Quality of Life assessment (WHOQOL) position paper from the World Health Organization. Soc Sci Med 41: 14031409.

41. Power M, Schmidt S, WHOQOL Group (2006) WHOQOL-OLD manual. World Health Organization

42. Straus MA, Hamby SL, BoneyMcCoy S, Sugarman DB (1996) The revised Conflict Tactics Scales (CTS2). Development and preliminary psychometric data. J Fam Issues 17: 283-316.

43. Brähler E, Scheer JW (1995) Der gießenerbeschwerdebogen (GBB) (2. Aufl) Bern: Huber.

44. Zigmond AS, Snaith RP (1983) The hospital anxiety and depression scale. Acta Psychiatr Scand 67: 361-370.

45. Folkhälsorapport (2007) Folkhälsan i Stockholmslän 2007 [In Swedish] Stockholm: Centrum förfolkhälsa, Stockholmslänslandsting

46. Zimet GD, Dahlem NW, Zimet SG, Farley GK (1988) The multidimensional scale of perceived social support. J Pers Assess 52: 30-41.

47. Babor TF, Higgins-Biddle JC, Saunders JB, Monteiro MG (2001) The Alcoho Use Disorders Identification Test: Guidelines for use in primary care. (2ndedn) Geneva: World Health Organization Department of Mental Health and Substance Dependence.

48. Kleinschmidt KC (1997) Elder abuse: a review. Ann Emerg Med 30: 463-472.

49. Luo Y, Waite LJ (2011) Mistreatment and psychological well-being among older adults: exploring the role of psychosocial resources and deficits. J Gerontol B Psychol Sci Soc Sci 66: 217-229.

50. Podkieks E (1992) National survey on abuse of the elderly in Canada. J Elder Abuse Neg 14: 5-58.

51. Gray A (2009) The social capital of older people. Ageing Soc 29: 5-31.

52. Stroebe W (2000) Moderators of the stress-health relationship. In Social psychology and health. Editor: Stroebe W, Philadelphia, PA: Open University Press.

53. Lachs MS, Williams C, O'Brien S, Hurst L, Horwitz R (1997) Risk factors for reported elder abuse and neglect: a nine-year observational cohort study. Gerontologist 37: 469-474.

54. Melchiorre MG, Chiatti C, Lamura G, Torres-Gonzales F, Stankunas M, et al (2013) Social support, socio-economic status, health and abuse among olde people in seven European countries. PLoS One 8: e54856.

55. Molzahn AE, Kalfoss M, Schick Makaroff K, Skevington SM (2011) Comparing the importance of different aspects of quality of life to older adults across diverse cultures. Age Ageing 40: 192-199.

56. Buber I, Kuhn M, Philipov D, Prskawetz A, Schuster J (2010) The economic situation of older cohorts in Europe. Vienna Institute of Demography of the Austrian Academy of Sciences.

57. Low G, Molzahn AE (2007) Predictors of quality of life in old age: a crossvalidation study. Res Nurs Health 30: 141-150.

58. Welford C, Murphy K, Wallace M, Casey D (2010) A concept analysis of autonomy for older people in residential care. J Clin Nurs 19: 1226-1235.

59. Haak M (2006) Participation and independence in old age - Aspects of home and neighbourhood environments. InstitutionenförHälsa, VårdochSamhälle, LundsUniversitet, Sverige.

60. Hwang HL, Lin HS, Tung YL, Wu HC (2006) Correlates of perceived autonomy among elders in a senior citizen home: a cross-sectional survey. Int $\mathrm{J}$ Nurs Stud 43: 429-437.

61. Matsui M, Capezuti E (2008) Perceived autonomy and self-care resources among senior center users. Geriatr Nurs 29: 141-147.

62. DeLamater J (2012) Sexual expression in later life: a review and synthesis. $J$ Sex Res 49: 125-141.

63. Frankowski AC, Clark LJ (2009) Sexuality and intimacy in assisted living: Residents' perspectives and experiences. Sex Res Social Policy 6: 25-37.

64. Lindau ST, Gavrilova N (2010) Sex, health, and years of sexually active life gained due to good health: evidence from two US population based cross sectional surveys of ageing. BMJ 340: c810.

65. Rheaume C, Mitty E (2008) Sexuality and intimacy in older adults. Geriatr Nurs 29: $342-349$

66. Robinson JG, Molzahn AE (2007) Sexuality and quality of life. J Gerontol Nurs 33: $19-27$.

67. Zaidi A, Makovec M, Fuchs M, Lipszyc B, Lelkes O, et al. (2006) Poverty of elderly people in EU25. Vienna: European Centre for Social Welfare Policy and Research.

68. Zaidi A (2010) Poverty risks for older people in EU countries - An Update. Policy Brief Series, Vienna: European Centre for Social Welfare Policy and Research.

69. Orth U, Trzesniewski KH, Robins RW (2010) Self-esteem development from young adulthood to old age: a cohort-sequential longitudinal study. J Pers Soc Psychol 98: 645-658

70. Robins RW, Trzesniewski KH (2005) Self-esteem development across the lifespan. Curr Dir Psychol Sci14: 158-162.

71. Fokkema T, De Jong Gierveld J, Dykstra PA (2012) Cross-national differences in older adult loneliness. J Psychol 146: 201-228.

72. Sundström G, Fransson E, Malmberg B, Davey A (2009) Loneliness among older Europeans. Eur J Ageing 6:267-275.

73. Kalfoss M, Halvorsrud L (2009) Important issues to quality of life among norwegian older adults: an exploratory study. Open Nurs J 3: 45-55.

74. Babatsikou F, Zavatsanou A (2010) Epidemiology of hypertension in the elderly. Health Sci J 4: 24-30. 
Citation: Soares JJF, Sundin Ö, Viitasara E, Melchiorre MG, Stankunas M, et al. (2013) Quality of Life among Persons Aged 60-84 Years in Europe: The Role of Psychological Abuse and Socio-Demographic, Social and Health Factors. J Biosafety Health Educ 1: 101. doi:10.4172/23320893.1000101

Page 11 of 12

75. Blazer DG (2009) Depression in late life: Review and commentary. Focus 7: $118-136$

76. Breivik H, Collett B, Ventafridda V, Cohen R, Gallacher D (2006) Survey of chronic pain in Europe: prevalence, impact on daily life, and treatment. Eur J Pain 10: 287-333.

77. Hawthorne G (2008) Perceived social isolation in a community sample: its prevalence and correlates with aspects of peoples' lives. Soc Psychiatry Psychiatr Epidemiol 43: 140-150.

78. Jemal A, Bray F, Center MM, Ferlay J, Ward E, et al. (2011) Global cancer statistics. CA Cancer J Clin 61: 69-90.

79. Lenze EJ, Wetherell JL (2011) A lifespan view of anxiety disorders. Dialogues ClinNeurosci 13: 381-399.

80. Shaw JE, Sicree RA, Zimmet PZ (2010) Global estimates of the prevalence of diabetes for 2010 and 2030. Diabetes Res Clin Pract 87: 4-14.

81. Iliffe S, Kharicha K, Harari D, Swift C, Gillmann G, et al. (2007) Health risk appraisal in older people 2: the implications for clinicians and commissioners of social isolation risk in older people. Br J Gen Pract 57: 277-282.

82. Lloyd-Jones D, Adams R, Carnethon M, De Simone G, Ferguson TB, et al (2009) Heart disease and stroke statistics--2009 update: a report from the American Heart Association Statistics Committee and Stroke Statistics Subcommittee. Circulation 119: 21-181.

83. James BD, Wilson RS, Barnes LL, Bennett DA (2011) Late-life social activity and cognitive decline in old age. J Int Neuropsychol Soc 17: 998-1005.

84. Kendler KS, Myers J, Prescott CA (2005) Sex differences in the relationship between social support and risk for major depression: a longitudinal study of opposite-sex twin pairs. Am J Psychiatry 162: 250-256.

85. Brockmann H, Klein T (2004) Love and death in Germany: The marital biography and its effect on mortality. J Marriage Fam 66: 567-581.

86. Di Tella R, MacCulloch RJ, Oswald AJ (2003)The macroeconomics of Happiness. Rev Econ Stat 85:809-827.

87. Diener E, Gohm C, Suh E, Oishi S (2000) Similarity of the relations between marital status and subjective well-being across cultures. J Cross Cul Psychol 31: 419-436.

88. Koball H, Moiduddin E, Henderson J, Goesling B, Besculides M (2010) What do we know about the link between marriage and health? J Fam Issues 31 1019-1040.

89. Schoenborn CA (2004) Marital Status and Health: United States, 1999-2002. Adv Data 15: 1-32

90. Wilson CM, Oswald AJ (2005) How does marriage affect physical and psychological health? A survey of the longitudinal evidence. IZA Discussion Paper No. 1619

91. Wood RG, Goesling B, Avellar S (2007) The effects of marriage on health A synthesis of recent research evidence. Princeton, NJ: Mathematica Policy Research.

92. Silverstein M, Gans D (2006) Intergenerational support to aging parents: The role of norms and needs. J Fam Issues 27: 1068-1084.

93. Turagabeci AR, Nakamura K, Kizuki M, Takano T (2007) Family structure and health, how companionship acts as a buffer against ill health. Health Qual Life Outcomes 5: 61 .

94. Ryan AK, Willits FK (2007) Family ties, physical health, and psychological wellbeing. J Aging Health 19: 907-920.

95. Guedes DP, Hatmann AC, Martini FA, Borges MB, Bernardelli R Jr (2012) Quality of life and physical activity in a sample of Brazilian older adults. J Aging Health 24: 212-226.

96. Bowles S, Durlauf S, Hoff K (2006) Poverty traps. Princeton University Press.

97. Lacey EA, Walters SJ (2003) Continuing inequality: gender and social class influences on self perceived health after a heart attack. J Epidemiol Community Health 57: 622-627.

98. Marmot M, Wilkinson R (2006) Social determinants of health, (2ndedn). Oxford: Oxford University Press.

99. Krause NM, Jay GM (1994) What do global self-rated health items measure? Med Care 32: 930-942.
100.Berkman L, Kawachi I (2000) Social epidemiology. New York: Oxford University Press.

101. Wilkinson R, Pickett K (2010) The spirit levelwhy more equal societies almost always do better. London: Penguin Books.

102. Al-Windi A (2005) The relations between symptoms, somatic and psychiatric conditions, life satisfaction and perceived health. A primary care based study. Health Qual Life Outcomes 3: 28.

103. Hoedeman R, Blankenstein AH, Krol B, Koopmans PC, Groothoff JW (2010) The contribution of high levels of somatic symptom severity to sickness absence duration, disability and discharge. J Occup Rehabil 20: 264-273.

104. Ihlebaek C, Eriksen HR, Ursin H (2002) Prevalence of subjective health complaints (SHC) in Norway. Scand J Public Health 30: 20-29.

105. Eurostat (2008) The life of women and men in Europe: A statistical portrait. Luxembourg: Eurostat.

106. Grundy E (2006)Ageing and vulnerable elderly people: European perspectives Ageing Soc 26: 105-134.

107. Winquist K (2002) Women and men beyond retirement. Statistics in focus population and social conditions, Luxembourg: Eurostat.

108. Chiao C, Weng LJ, Botticello AL (2012) Economic strain and well-being in late life: findings from an 18-year population-based Longitudinal Study of older Taiwanese adults. J Public Health (Oxf) 34: 217-227.

109. Fryers T, Melzer D, Jenkins R (2003) Social inequalities and the common mental disorders: a systematic review of the evidence. Soc Psychiatry Psychiatr Epidemiol 38: 229-237.

110. Hall SE, Williams JA, Senior JA, Goldswain PR, Criddle RA (2000) Hip fracture outcomes: quality of life and functional status in older adults living in the community. Aust N Z J Med 30: 327-332.

111. Ladin K, Daniels N, Kawachi I (2010) Exploring the relationship between absolute and relative position and late-life depression: evidence from 10 European countries. Gerontologist 50: 48-59.

112. Mikulic B, Sándor E, Leoncikas T (2012) Experiencing the economic crisis in the EU: Changes in living standards, deprivation and trust. European Foundation for the Improvement of Living and Working Conditions.

113. Sampaio PY, Ito E (2013) Activities with higher influence on quality of life in older adults in Japan. Occup Ther Int 20: 1-10.

114. Paskulin L, Vianna L, Molzahn AE (2009) Factors associated with quality of life of Brazilian older adults. Int Nurs Rev 56: 109-115.

115. Blood R, Wolfe D (1960) Husbands and wives: The dynamics of married living New York: Free Press.

116. Ferree MM (1990) Beyond separate spheres: Feminism and family research J Marriage Fam 52: 866-884

117. Pahl J (1990) Household spending, personal spending and the control of money in marriage. Sociology 24: 119-138.

118. Brennan RT, Barnett RC, Gareis KC (2001) When she earns more than he does: A longitudinal study of dual-earner couples. J Marriage Fam 63: 168182.

119. Pappenheim H, Graves $\mathrm{G}$ (2005) Bringing home the bacon: Making marriage work when she makes more money. New York: William Morrow.

120. Umberson D, Williams K, Powers DA, Liu H, Needham B (2006) You make me sick: marital quality and health over the life course. J Health Soc Behav 47: 1-16.

121. Springer KW (2010) Do wives' work hours hurt husbands' health? Reassessing the care work deficit thesis. Soc Sci Res 39: 801-813.

122. Anderson P, Baumberg B (2006) Alcohol in Europe. London: Institute of Alcohol Studies.

123. Holahan CJ, Schutte KK, Brennan PL, Holahan CK, Moos BS, et al. (2010) Late-life alcohol consumption and 20-year mortality. Alcohol Clin Exp Res 34: 1961-1971.

124. Kaplan MS, Huguet N, Feeny D, McFarland BH, Caetano R, et al. (2012) Alcohol use patterns and trajectories of health-related quality of life in middleaged and older adults: a 14-year population-based study. J Stud Alcohol Drugs 73: 581-590. 
Citation: Soares JJF, Sundin Ö, Viitasara E, Melchiorre MG, Stankunas M, et al. (2013) Quality of Life among Persons Aged 60-84 Years in Europe: The Role of Psychological Abuse and Socio-Demographic, Social and Health Factors. J Biosafety Health Educ 1: 101. doi:10.4172/23320893.1000101

125. Paganini-Hill A, Kawas $\mathrm{CH}$, Corrada MM (2007) Type of alcohol consumed changes in intake over time and mortality: the Leisure World Cohort Study. Age Ageing 36: 203-209.

126. Bowling A, lliffe S (2011) Psychological approach to successful ageing predicts future quality of life in older adults. Health Qual Life Outcomes 9: 13.

127. de Belvis AG, Avolio M, Spagnolo A, Damiani G, Sicuro L, et al. (2008) Factors associated with health-related quality of life: the role of social relationships among the elderly in an Italian region. Public Health 122: 784-793.

128. George LK (2006) Perceived Quality of Life. In Handbook of aging and the social sciences.(6thedn), Editors: Binstock RH, George LK. San Diego, CA: Elsevier.

129. White AM, Philogene GS, Fine L, Sinha S (2009) Social support and selfreported health status of older adults in the United States. Am J Public Health 99: $1872-1878$

130. Martinez-Martin P, Prieto-Flores M, Forjaz MJ, Fernandez-Mayorales G, Rojo-Perez F, et al. (2012) Components and determinants of quality of life in community-dwelling older adults. Eur J Ageing 9: 255-263.
131. Cohen S, Wills TA (1985) Stress, social support, and the buffering hypothesis Psychol Bull 98: 310-357.

132. Gureje O, Kola L, Ademola A, Olley BO (2009) Profile, comorbidity and impact of insomnia in the Ibadan study of ageing. Int J Geriatr Psychiatry 24: 686-693.

133. LeBlanc M, Mérette C, Savard J, Ivers H, Baillargeon L, et al. (2009) Incidence and risk factors of insomnia in a population-based sample. Sleep 32: 10271037.

134. Faubel R, Lopez-Garcia E, Guallar-Castillón P, Balboa-Castillo T, GutiérrezFisac JL, et al. (2009) Sleep duration and health-related quality of life among older adults: a population-based cohort in Spain. Sleep 32: 1059-1068.

135. Lo CM, Lee PH (2012) Prevalence and impacts of poor sleep on quality of life and associated factors of good sleepers in a sample of older Chinese adults. Health Qual Life Outcomes 10: 72 